\title{
The range of validity of cluster masses and ages derived from broad-band photometry
}

\author{
J. Maíz Apellániz \\ Instituto de Astrofísica de Andalucía-CSIC, Granada, Spain \\ email: jmaiz@iaa.es
}

\begin{abstract}
I analyze the stochastic effects introduced by sampling the stellar initial mass function (SIMF) in the derivation of the individual masses and the cluster mass function (CMF) from broad-band visible/near-infrared unresolved photometry. The classical method of using unweighted $U B V$ photometry to simultaneously establish ages and extinctions of stellar clusters is found to be unreliable for clusters older than $\approx 30 \mathrm{Myr}$, even for relatively large cluster masses. On the other hand, augmenting the filter set to include longer-wavelength filters and using weights for each filter increases the range of masses and ages that can be measured accurately with unresolved photometry. Nevertheless, a relatively large range of masses and ages is found to be dominated by SIMF sampling effects that render the observed masses useless, even when using $U B V R I J H K$ photometry.
\end{abstract}

Keywords. methods: analytical, methods: numerical, methods: statistical, open clusters and associations: general, globular clusters: general, galaxies: star clusters

\section{Description}

This work is the third in a series in which we are analyzing the possible biases present in mass functions (Maíz Apellániz \& Úbeda 2005; Maíz Apellániz 2008). I have used a combination of analytical approximations and Monte Carlo simulations to study the effect of stochastically sampling the stellar IMF (SIMF; assumed to be of Kroupa type) in the determination of masses and ages of unresolved stellar custers from broad-band photometry. For this purpose, I have used the new version (3.1) of CHORIzos (Maíz Apellániz 2004), which incorporates an evolutionary synthesis module. For a given mass and age, I generated a minimum of 10000 realizations of the SIMF using solar-metallicity (a) Geneva isochrones for massive stars and (b) Padova isochrones with new asymptotic giant branch (AGB) treatment for low and intermediate masses. In each case, I measured the mass (and age, if appropriate) assuming that the cluster has a well-sampled SIMF. From the individual realizations I derived (a) the observed cluster mass and age distributions for clusters of a (fixed) real mass and age and (b) the observed cluster mass functions (CMFs) for a real, truncated-power-law CMF with a slope of $\gamma=-2.0$. Three different cases in order of complexity were considered: (i) single-filter observations of clusters with known age and extinction, (ii) multifilter observations of clusters with unknown age and known extinction and (iii) multifilter observations of clusters with unknown age and extinction. In the first case, masses were computed directly by converting filter-convolved luminosities to masses, while in the second and third cases a $\chi^{2}$ minimization code (CHORIzOs) was used to derive the masses and ages. The reader is referred to Maíz Apellániz (2009) for further details. 

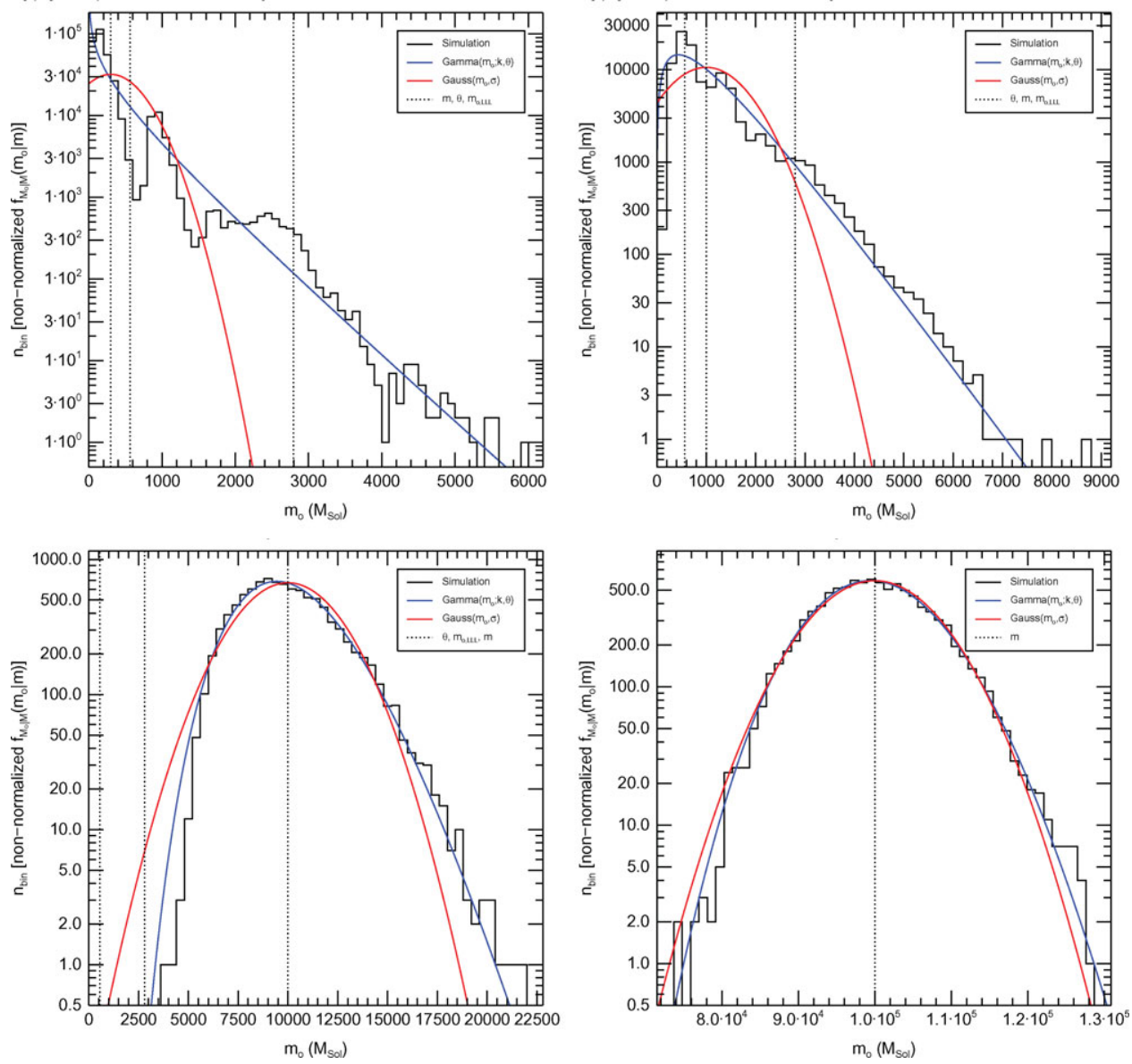

Figure 1. Results of the Monte Carlo simulations of $f_{M_{\mathrm{o}} \mid M}\left(m_{\mathrm{o}} \mid m\right)$ and Gaussian and Gamma fits for 10 Myr-old clusters observed in $V$ with four real masses: $300 \mathrm{M}_{\odot}$ (top left), $1000 \mathrm{M}_{\odot}$ (top right), $10000 \mathrm{M}_{\odot}$ (bottom left) and $100000 \mathrm{M}_{\odot}$ (bottom right). The Poisson mass $\theta$ for this filter and age is $566 \mathrm{M}_{\odot}$. For large real masses, both fits provide reasonable approximations, but for small values of $m$ the Gamma approximation is significantly better. The vertical dotted lines show the values of the real mass, $m$, and (when visible) of $\theta$ and $m_{\mathrm{o}, \mathrm{LLL}}$ (the observed cluster mass obtained when one mistakes the brightest possible star in the isochrone for a cluster).

\section{Case 1: single filter, known age and extinction}

- For a given cluster mass $(m)$, the observed cluster mass $\left(m_{\mathrm{o}}\right)$ distribution can be better approximated by a Gamma function than by a Gaussian distribution (Figure 1):

$$
f_{M_{\mathrm{o}} \mid M}\left(m_{\mathrm{o}} \mid m\right)=A m_{\mathrm{o}}^{m / \theta-1} e^{-m_{\mathrm{o}} / \theta} \theta^{-m / \theta} .
$$

- The Poisson mass, $\theta$, is a measurement of the stochasticity and depends (strongly) on the age and the filter used to derive $m_{\mathrm{o}}$ (Table 2 ).

$$
\overline{m_{\mathrm{o}}}=m \quad ; \quad \sigma_{m_{\mathrm{o}}}=\sqrt{\theta m} .
$$

- The large differences in $\theta$ are a consequence of the different fractions of stars located above the median mass for the luminosity in each filter, for a given isochrone (Figure 2).

- The observed CMF, $f_{M_{\mathrm{o}}}\left(m_{\mathrm{o}}\right)$, differs from the real (truncated power-law) CMF, $f_{M}(m)$, in two aspects (Figure 3): (i) For large masses, $f_{M_{\mathrm{o}}}\left(m_{\mathrm{o}}\right)$ is similar to $f_{M}(m)$ but, 


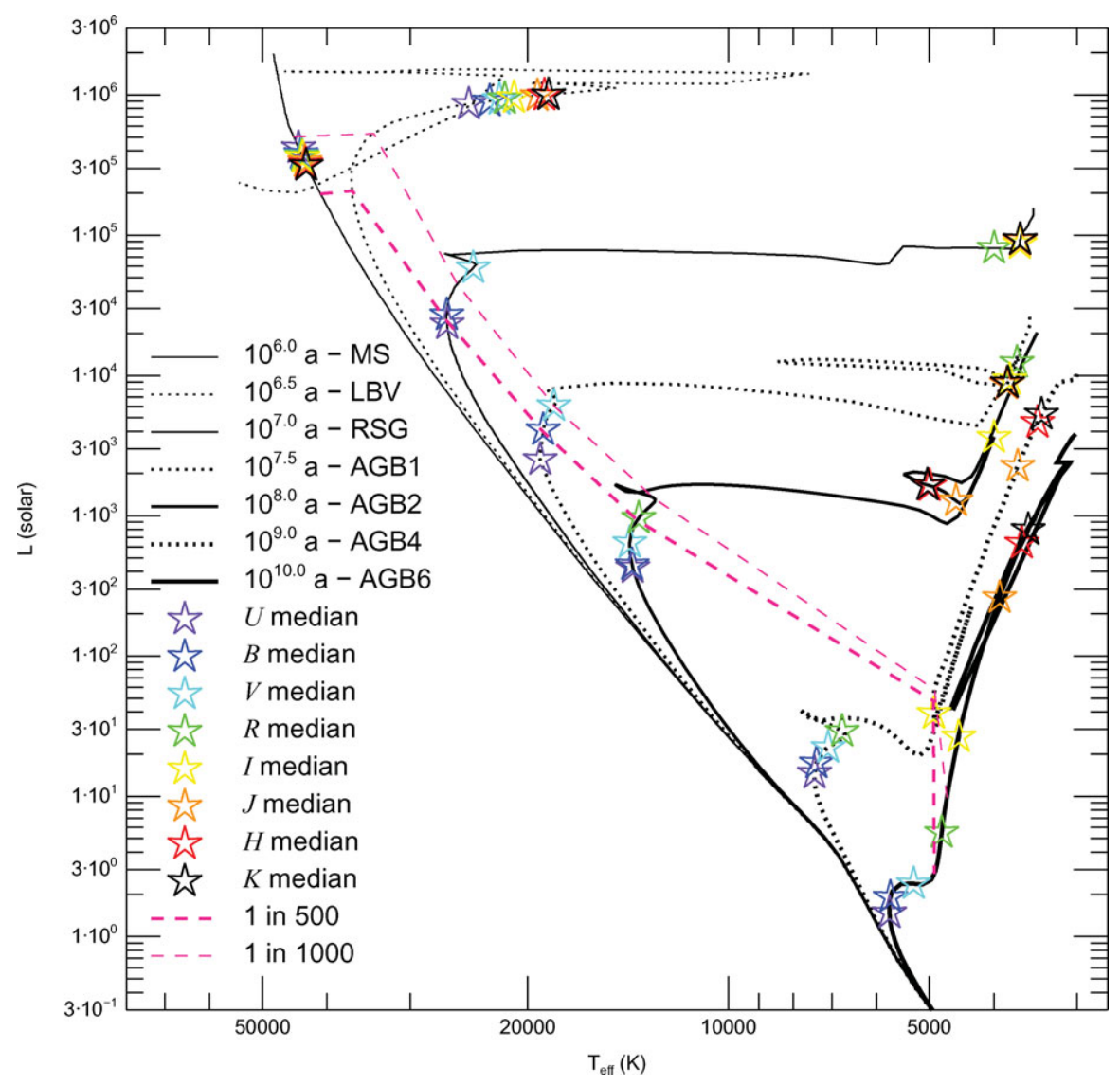

Figure 2. Isochrones for seven of the nine ages used in this work. For each isochrone, symbols of different colors are used to indicate the median mass for the luminosity in the eight filters $U, B$, $V, R, I, J, H$ and $K$, assuming a Kroupa SIMF between 0.1 and $120 \mathrm{M}_{\odot}$. The two dashed lines join the points in each isochrone beyond which 0.2 and $0.1 \%$, respectively, of the remaining stars in a well-sampled Kroupa SIMF are located. The two remaining isochrones are not included for the sake of clarity.

as the cluster mass decreases, at one point a 'hump' or overdensity appears. (ii) To the left of the hump, $f_{M_{\mathrm{o}}}\left(m_{\mathrm{o}}\right)$ asymptotically approaches a power law with a slope greater than -1.0 , with some clusters having $m_{\mathrm{o}}$ values lower than the real cutoff in $m$.

- Using an analytical approximation, it can be shown that if one is willing to tolerate a systematic error in the CMF slope $\gamma$ of up to $\Delta \gamma$, one can only analyze observed masses

$$
m_{\mathrm{o}}>0.5 \gamma(\gamma+1) \theta / \Delta \gamma
$$

e.g., for $\gamma=-2.0$ and $\Delta \gamma=0.1, m_{\mathrm{o}}>10 \theta$.

\section{Case 2: multiple filters, unknown age, and known extinction}

I used three execution types:

1. $U B V R I J H K$ photometry with single-band $\theta$-dependent weights for each filter;

2. $U B V R I J H K$ photometry with constant weights;

3. $U B V$ photometry with single-band $\theta$-dependent weights for each filter. 
Table 1. Values of $\theta$ (in $\mathrm{M}_{\odot}$ ) for different ages and filters for clusters observed with a single filter and known age and extinction. The labels below each age indicate the type of the brightest possible star. For a given filter, $\theta$ starts at a low value in the main-sequence (MS) phase, grows rapidly in the luminous-blue-variable (LBV) phase, decreases during the red-supergiant (RSG) and early AGB phases and then experiences a moderate growth when the cluster becomes old. For a given age, $\theta$ increases as a function of wavelength with the only exception of the MS phase, where the effect is the opposite (but rather weak).

\begin{tabular}{crrrrrrrrr}
\hline \multirow{2}{*}{ Filter } & $\begin{array}{r}1 \mathrm{Myr} \\
\text { MS }\end{array}$ & $\begin{array}{r}3.16 \mathrm{Myr} \\
\text { LBV }\end{array}$ & $\begin{array}{r}\text { 10 Myr } \\
\text { RSG }\end{array}$ & $\begin{array}{r}31.6 \mathrm{Myr} \\
\text { AGB1 }\end{array}$ & $\begin{array}{r}100 \mathrm{Myr} \\
\text { AGB2 }\end{array}$ & $\begin{array}{r}316 \mathrm{Myr} \\
\text { AGB3 }\end{array}$ & $\begin{array}{r}\text { AGyr } \\
\text { AGB }\end{array}$ & $\begin{array}{r}\text { 3.16 Gyr } \\
\text { AGB5 }\end{array}$ & $\begin{array}{r}\text { 10 Gyr } \\
\text { AGB6 }\end{array}$ \\
\hline$U$ & 235 & 945 & 160 & 149 & 53 & 33 & 23 & 26 & 42 \\
$B$ & 200 & 1314 & 192 & 305 & 51 & 32 & 27 & 52 & 92 \\
$V$ & 190 & 1555 & 566 & 370 & 89 & 38 & 51 & 106 & 163 \\
$R$ & 186 & 1722 & 1034 & 443 & 151 & 72 & 109 & 173 & 242 \\
$I$ & 178 & 1949 & 1585 & 616 & 273 & 463 & 476 & 477 & 581 \\
$J$ & 157 & 2741 & 2240 & 1170 & 708 & 3598 & 2930 & 2702 & 2445 \\
$H$ & 146 & 3081 & 2476 & 1640 & 1281 & 5184 & 4828 & 4499 & 4123 \\
$K$ & 140 & 3333 & 2542 & 1771 & 1527 & 5717 & 5688 & 5512 & 5058 \\
\hline
\end{tabular}
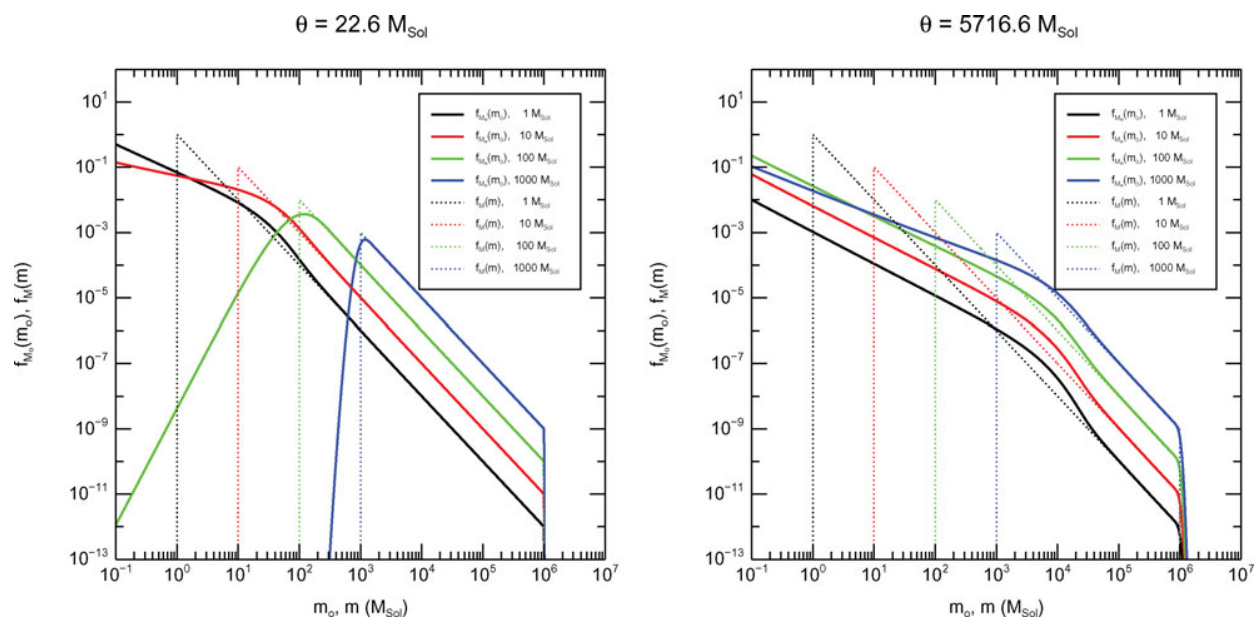

Figure 3. Observed (continuous lines) and real (dotted lines) CMFs for the minimum and maximum values of $\theta$ in Table 2. The left panel corresponds to 1 Gyr-old clusters observed in $U$ and the right panel to 316 Myr-old clusters observed in $K$. Each panel shows four cases of the lower mass cutoff for $f_{M}(m)$ from 1 to $1000 \mathrm{M}_{\odot}$. As $\theta$ increases, the hump becomes more pronounced and moves towards the right.

The observed age distributions for $10^{4} \mathrm{M}_{\odot}$ clusters are shown in Figure 4 . The main results are:

- The ages calculated with $U B V R I J H K$ photometry and constant weights (i.e., the standard method) are highly uncertain, even for $10^{4} \mathrm{M}_{\odot}$ clusters.

- Using weights based on the stochasticity of each filter significantly narrows the observed age distribution for $10^{4} \mathrm{M}_{\odot}$ clusters, thus allowing to obtain ages with relatively small uncertainties.

- $U B V$ and $U B V R I J H K$ photometry provide relatively similar results, with the former having the advantage for old clusters and the latter for their intermediate-age counterparts. 

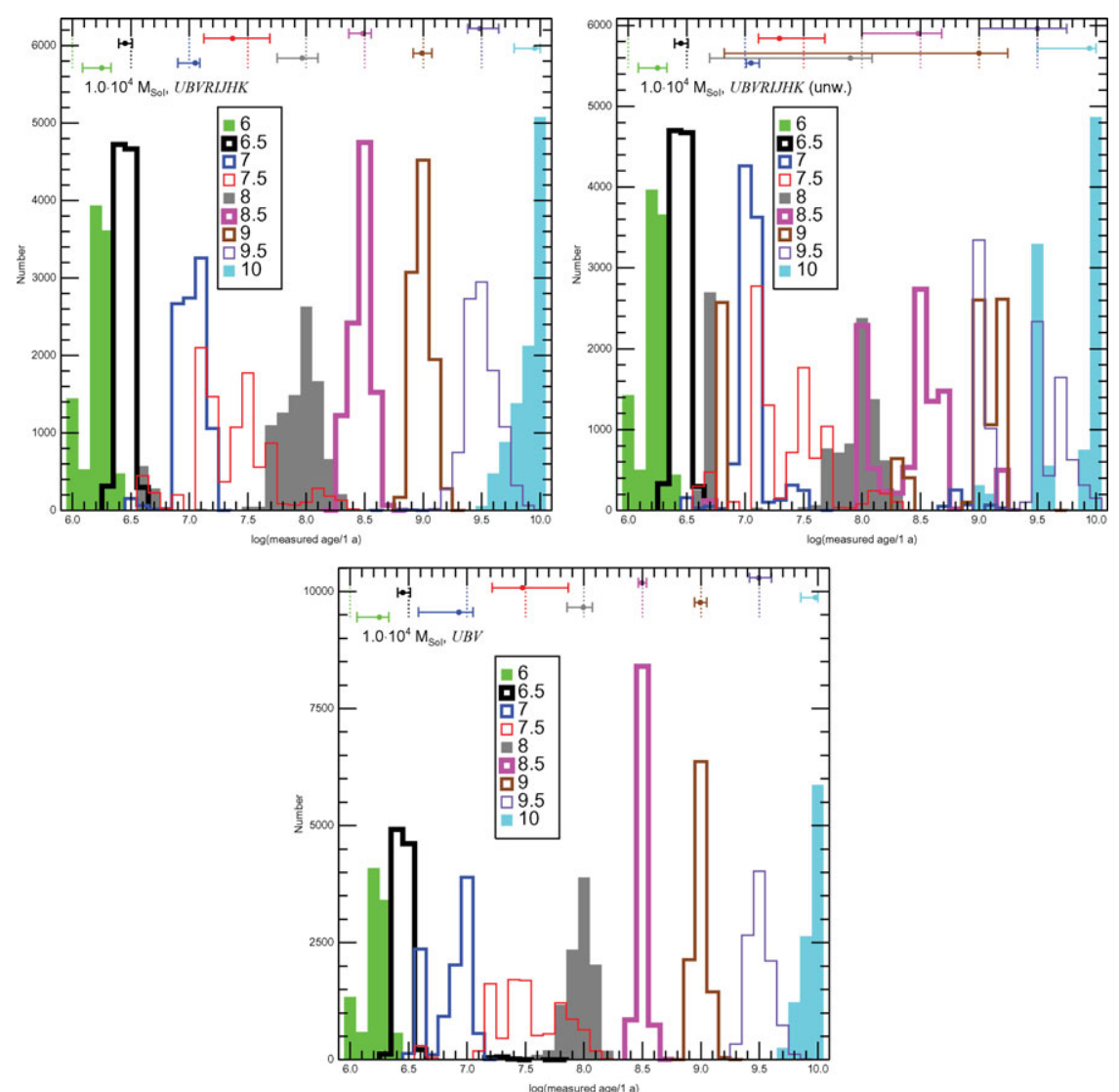

Figure 4. Distribution of observed ages for the nine input ages and three execution types (1: upper left, 2: upper right, 3: bottom) for clusters of $10^{4} \mathrm{M}_{\odot}$ and unknown ages. At the top of each panel, the dashed line indicates the real age while the symbols and error bars provide the median and inferior and superior uncertainties ( $1 \sigma$ equivalents) of each distribution.

- The AGB1 stage (31.6 Myr) has significantly greater uncertainties than the other evolutionary phases.

\section{Case 3: multiple filters, unknown age and extinction}

I used two execution types:

1. $U B V R I J H K$ photometry with single-band $\theta$-dependent weights for each filter;

2. $U B V$ photometry with single-band $\theta$-dependent weights for each filter.

The observed age distributions for $10^{4}$ and $10^{5} \mathrm{M}_{\odot}$ clusters are shown in Figure 5 . The main results are:

- As opposed to the cases where extinction is known, the addition of RIJHK photometry provides a significant improvement in the observed ages.

- $U B V$ photometry alone is not sufficient to accurately determine the ages of unresolved stellar clusters, even for massive ones, if extinction is unknown.

- For some ages (1-10 Myr, $100 \mathrm{Myr}, 10 \mathrm{Gyr})$, UBVRIJHK photometry provides relatively accurate observed ages for $m=10^{4} \mathrm{M}_{\odot}$. For other ages, higher masses are needed. 

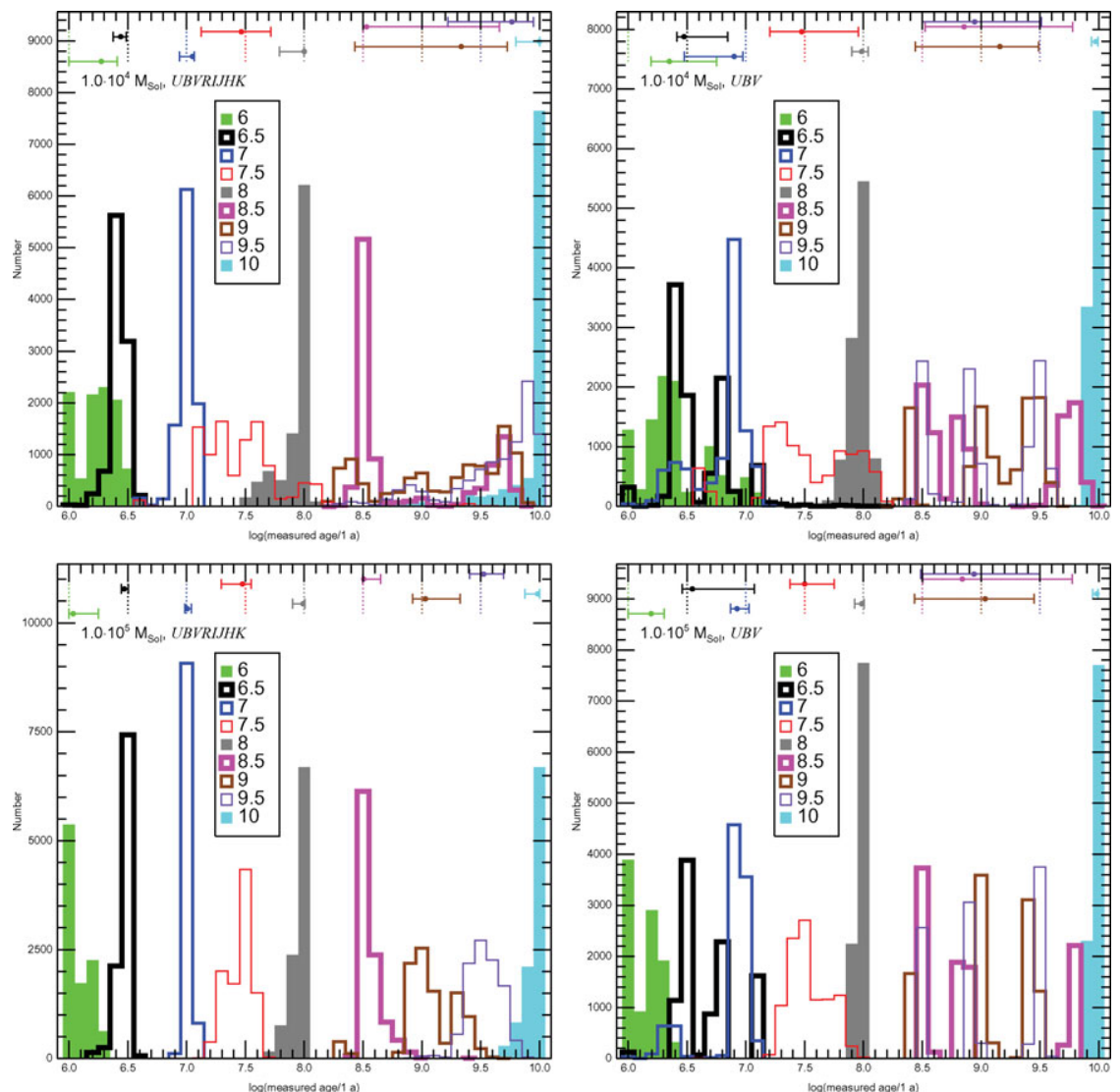

Figure 5. Distribution of observed ages for the nine input ages and two execution types (1: left plots, 2: right plots) for clusters of $10^{4}$ (top) and $10^{5} \mathrm{M}_{\odot}$ (bottom) and unknown ages and extinctions. At the top of each panel, the dashed line indicates the real age, while the symbols and error bars provide the median and inferior and superior uncertainties ( $1 \sigma$ equivalents) of each distribution.

\section{Conclusions}

- SIMF sampling effects can introduce large biases in the determination of masses and ages from unresolved photometry.

- One should use weights based on the relative stochasticity of each filter when using $\chi^{2}$-minimization techniques to calculate cluster properties from unresolved photometry.

- The addition of a wide photometric baseline ( $U$ to $K$ ) significantly reduces uncertainties when extinction is determined from the data.

- For clusters younger than $100 \mathrm{Myr}$, a critical (post-RSG) stage exists around $30 \mathrm{Myr}$ where ages are especially difficult to determine.

\section{References}

Maíz Apellániz, J. 2004, PASP, 116, 859

Maíz Apellániz, J. 2008, ApJ, 677, 1278

Maíz Apellániz, J. 2009, ApJ, 699, 1938

Maíz Apellániz, J. \& Úbeda, L. 2005, ApJ, 629, 873 